This study quantifies the risk of hospitalisation relating to overweight and obesity.

Methods 24194945 and Up Study participants with linked hospital admissions and death data were followed from recruitment (February 2006 onwards) through to June 2009. Self-reported height and weight were used to classify patients into BMI categories, using cut-points of 15, 18.5, 20, 22.5, 25, 27.5, 30 and $35 \mathrm{~kg} / \mathrm{m}^{2}$. Rates of incident hospitalisation by BMI were compared using Cox regression, adjusting for a range of confounders.

Results Preliminary results on the first 103040 participants show incident hospitalisation rates to be 299 (95\% CI 294 to 304) per 1000 person years for males and 248 (95\% CI 243 to 252) for females. Compared with those of healthy-weight (BMI $20-22.5 \mathrm{~kg} / \mathrm{m}^{2}$ ), rates in those with severe obesity (BMI $35-50 \mathrm{~kg} / \mathrm{m}^{2}$ ) were higher among males (HR: $1.36,95 \%$ CI 1.21 to 1.54 ) and females (HR: 1.52; $95 \%$ CI 1.38 to 1.67). There were clear gradients as weight increased from healthy to higher BMI, more so among females than males, and in people aged 45-64 compared to those aged 65-84, with no evidence of increasing risk of hospitalisation with increasing BMI in those aged over 85 .

Conclusions Given the excess risk of hospitalisation among overweight and obese individuals, the burden to the health system attributable to overweight and obesity is likely to be substantial, particularly among middle-age adults. High BMI was not significantly related to hospitalisation in the elderly.

\section{P2-146 EPIDEMIOLOGICAL AND CLINICAL CHARACTERISTICS OF BEHÇET'S DISEASE IN JAPAN, BY YEARS AFTER DISEASE ONSET, USING A CLINICAL DATABASE ON PATIENTS RECEIVING FINANCIAL AID FOR TREATMENT}

doi:10.1136/jech.2011.142976i.81

\begin{abstract}
${ }^{1} \mathrm{M}$ Kurosawa, ${ }^{* 1,2} \mathrm{Y}$ Inaba, ${ }^{3} \mathrm{Y}$ Ishigatsubo, ${ }^{3} \mathrm{M}$ Takeno, ${ }^{4} \mathrm{M}$ Nagai, ${ }^{1} \mathrm{~K}$ Yokoyama. ${ }^{1}$ Department of Epidemiology and Environmental Health, Juntendo University Faculty of Medicine, Bunkyo-Ku, Tokyo, Japan; '2Jissen Women's University, Hino, Tokyo, Japan; ${ }^{3}$ Department of Internal Medicine and Clinical Immunology, Yokohama City University Graduate School of Medicine, Yokohama, Kanagawa, Japan; ${ }^{4}$ Department of Public Health, Faculty of Medicine, Saitama Medical University, Iruma-gun, Saitama, Japan
\end{abstract}

Objective Behçet's disease is an autoimmune disease with multisystem vasculitis. The objective of this study was to explore the natural history of Behçet's disease after onset, using a clinical database on patients receiving financial aid for treatment.

Methods In the fiscal year 2005, 16627 patients with Behçet's disease were registered to receive public financial aid from the Ministry of Health, Labour and Welfare (MHLW) in Japan. The MHLW has an on-line registration system of intractable diseases including Behçet's disease. We obtained the 2005 clinical database, which contained 9416 patients with Behçet's disease. We confirmed the distribution of years from disease onset, and calculated duration from onset to the first doctor's visit. We analysed changes in disease severity, and prevalence of the types of Behçet's disease, according to years after disease onset.

Results The proportion of years from disease onset of less than 1 year was 9\%, 2-5 years was $15 \%$, 6-15 years was $30 \%$, and more than 16 years was $46 \%$. The average duration from onset to the first doctor's visit was about 2.5-3 years. Prevalence of the complete type of Behçet's disease increased with the years after disease onset. Regarding disease severity, the proportion of severe cases increased with the years from disease onset.

Conclusion Using a clinical database with Behçet's disease, we characterised the clinical/epidemiological features of Behçet's disease according to years after disease onset.
Funding This study was supported by a Grant-in-Aid for Research Committee of Intractable disease, provided by the MHLW, Japan.

\section{P2-147 CANCER MORTALITY AND INCIDENCE RISK ACCORDING TO SOCIOECONOMIC STATUS, A PROSPECTIVE STUDY IN KOREA}

doi:10.1136/jech.2011.142976i.82

${ }^{1,2}$ S-S Kweon, ${ }^{2} \mathrm{M}-\mathrm{H}$ Shin, ${ }^{2} \mathrm{~J}$ Choi. ${ }^{1} J e o n n a m$ Regional Cancer Center, Chonnam National University Hwasun Hospital, Hwasun, Jeonnam, Republic of Korea; ${ }^{2}$ Chonnam National University Medical School, Department of Preventive Medicine, Dong-gu, Gwangju, Republic of Korea

Introduction The aim of this study was to evaluate the risk of socioeconomic status (SES) and smoking on cancer mortality and incidence in a large cohort of health examinee.

Methods We evaluated data on 512713 Korean people who had undergone the biannual health examination, organised by National Health Insurance Corporation, aged over 20 at baseline examination. Subjects were classified into four groups according to their amount of health insurance bill, which imposed in proportion to salary or income. All subjects were followed up from baseline examination (2000-2001) until 31 December 2009 using population-based cancer registry and death certification database. A total of 9166 cancer death cases and 27792 cancer incident cases were identified during follow-up period. Cox proportional hazards model was used to estimate hazard risk (HR) after adjusted age, sex, and smoking status.

Results SES had inverse associations with cancer mortality. The estimated HRs ( $95 \%$ CI) were 0.968 (0.916 to 1.023 ), 0.939 (0.877 to $0.985)$, and $0.790(0.746$ to 0.837$)$ in $2^{\text {nd }}-4^{\text {th }}$ Quartile, respectively. Smoking habits showed higher risk of cancer death (HR: 1.319, 95\% CI 1.259 to 1.382 ) than lower SES, and also showed significant association with cancer incidence (HR: 1.136, 95\% CI 1.101 to 1.172). The association between SES and cancer incidence showed positive trend as opposed to cancer mortality. The highest SES group were at greatest risk of cancer incidence (HR: 1.136, 95\% CI 1.096 to $1.176)$, and $2^{\text {nd }} \mathrm{Q}$ and $3^{\text {rd }} \mathrm{Q}$ group were also showed significant higher risk.

Conclusion Impact of SES on the risk of cancer mortality and incidence were showed reversely.

\section{P2-148 EARLY LIFE INFECTIONS AND PUBERTAL ONSET: EVIDENCE FROM HONG KONG'S “CHILDREN OF 1997" BIRTH COHORT}

doi:10.1136/jech.2011.142976i.83

M K Kwok, ${ }^{*}$ G M Leung, T H Lam, C M Schooling. Life course and Lifestyle Epidemiology Group, School of Public Health, Li Ka Shing Faculty of Medicine, The University of Hong Kong, Hong Kong SAR, China

Introduction With economic development, puberty occurs at younger ages, and may contribute to cardiovascular diseases and hormone-related cancers. Factors determining pubertal timing are poorly understood. The growth axis active during puberty is also active in the first 6 months of life and interacts with the immune system.

Methods The authors examined whether prior infections, proxied by number of hospital episodes for infections at different ages, were associated with age at pubertal onset (Tanner stage II) using interval-censored regression in a population-representative Chinese birth cohort "Children of 1997" ( $\mathrm{n}=7527)$. Mediation by growth was also examined. 
Results Girls, but not boys, hospitalised for infections at least twice in the first 6 months of life had pubertal onset at about 8 months older (mean 10.3 years), time ratio 1.08 (95\% CI: 1.04 to 1.12), than those without such hospitalisations (9.6 years) adjusted for infant characteristics and socio-economic position (sex interaction $\mathrm{p}$ value 0.02 ). There were no such associations for infections at 6 months to $<8$ years. Growth did not mediate the association.

Conclusion Early infectious morbidity in girls may be associated with later puberty, perhaps via suppression of the gonadotropic axis. Fewer infections in early life with economic development may be an additional factor contributing to earlier puberty.

\section{P2-149 BEHAVIOURAL RISK FACTORS FOR CVD AMONG ADULT SLUM DWELLERS IN NAIROBI}

doi:10.1136/jech.2011.142976i.84

\begin{abstract}
C Kyobutungi, ${ }^{*}$ S Oti. African Population and Health Research Center, Nairobi, Kenya
\end{abstract}
Introduction About $80 \%$ of deaths from non-communicable diseases (NCD) occur in developing countries and behavioural risk factors that underlie the burden of major NCD are on the rise in SSA. However, most research and development work has focused on communicable diseases such as malaria and HIV/AIDS while neglecting NCD. This study aims to contribute to the state of knowledge on the prevalence of modifiable risk factors for NCD specifically cardiovascular diseases in the adult population of two slums in Nairobi, Kenya.

Methods A cross-sectional survey among 5190 adults selected by stratified random sampling in two Nairobi slums covered. Data were collected on behavioural risk factors for cardiovascular diseases including self-reported tobacco and alcohol use, dietary habits and physical activity.

Results About $19 \%$ of respondents had ever used tobacco, and $12 \%$ were current users. Males were 20 times more likely to be current tobacco users than females. Majority of respondents (85\%) reported sufficient physical activity though large sex differences were observed. Females were three times more likely to be inactive than males. Less than half of respondents consumed sufficient fruits and vegetables while a little over a third had high salt consumption. Ten percent of respondents were current alcohol users of which, more than a third were frequent heavy drinkers.

Conclusion We found high levels of behavioural risk factors for NCD, specifically high alcohol and tobacco use and poor dietary habits albeit with marked gender differentials. Targeted intervention programmes that promote healthy lifestyles among the urban poor are recommended.

\section{P2-150 RESULTS OF THE 22-YEARS STUDY OF LEUKAEMIA IN CHERNOBYL ACCIDENT CLEAN-UP WORKERS IN UKRAINE}

doi:10.1136/jech.2011.142976i.85

A Romanenko, B Ledoshchuk. * Research Center for Radiation Medicine, Kyiv, Ukraine

Introduction The study was conducted to determine whether the leukaemia incidence rate in Chernobyl Nuclear Power Plant catastrophe clean-up workers depended on the year of the emergency work and the period after irradiation.

Methods All cases collected were verified. The analysis was performed for workers in 1986 and 1987 by periods of observation of 1987-1991 and 1992-1996.

Results Leukaemia RR in workers of 1986 vs workers of 1987 in 1987-1991 was 2.32 (95\% CI 1.19 to 4.70). No significant differences were identified in 1992-1996. The results of these studies were the beginning of the project "Leukaemia" in collaboration with the Cancer Institute of the U.S. The project used modern methods for epidemiological studies and created a database of case and controls. Further analysis is based on 71 cases of histologically confirmed leukaemia diagnosed in 1986-2000 and 501 residencematched controls selected from the same cohort Study subjects or their proxies were interviewed about their clean-up activities and other relevant factors. The excess RR (ERR) of total leukaemia was 3.44 per Gy (95\% CI 0.47 to $9.78, \mathrm{p}<0.01]$. We found a similar doseresponse relationship for chronic and non-chronic lymphocytic leukaemia $[E R R=4.09$ per Gy $(95 \% \mathrm{CI}<0$ to 14.41$)$ and 2.73 per Gy (95\% CI $<0$ to 13.50 ), respectively]. We found a linear dose-response relationship between Chernobyl-related radiation exposure among cleanup workers and their risk of leukaemia.

Conclusion The project plans to extend the case-control study to ascertain cases for another 6 years (2001-2006).

\section{P2-151 DETERMINANTS OF INCIDENT AND PERSISTENT FINGER JOINT PAIN DURING A 5-YEAR FOLLOW-UP AMONG FEMALE DENTISTS AND TEACHERS}

doi:10.1136/jech.2011.142976i.86

H Ding, S Solovieva, P Leino-Arjas. ${ }^{*}$ Finnish Institute of Occupational Health, Helsinki, Finland

Introduction To investigate the association of radiographic finger osteoarthritis $(\mathrm{OA})$, hand use, and lifestyle factors with incident and persistent finger joint pain among female dentists and teachers.

Methods Random samples of female dentists $(n=295)$ and teachers $(n=248)$ aged $45-63$ years were examined for the presence of finger joint $\mathrm{OA}$ by radiography. Body weight was measured. Information on finger joint pain during the past 30 days, height, smoking, and leisure-time hand activity was collected by questionnaire. Five years later, 482 women (ie, $89 \%$; $65 \%$ still occupationally active) responded to a survey on finger joint pain.

Results The incidence and persistence of finger joint pain were higher among the subjects with OA compared to those without. The RR of incident pain in the $1-3^{\text {rd }}$ fingers was 1.8 (95\% CI 1.2 to 2.7) in the right hand and 3.0 (2.0 to 4.6) in the left, allowing for age, occupation, and lifestyle factors. The corresponding figures for the $4-5^{\text {th }}$ fingers were 2.3 (1.4 to 3.8) in the right and 1.9 (1.1 to 3.5 ) in the left hand. Regarding persistent pain, the RRs varied between 2.4 and 5.4. Body mass index, smoking, or leisure-time hand activity were not associated with pain. The dentists tended to have a higher incidence of pain in the $1-3^{\text {rd }}$ fingers of the right hand than the teachers.

Conclusion Radiographic finger joint $\mathrm{OA}$ was a significant determinant of both persistent and incident finger joint pain in a 5-year follow-up among middle-aged women. Hand use may modify the association between radiographic $\mathrm{OA}$ and finger joint pain.

\section{P2-152 ASSESSING MENTAL HEALTH AMONG WOMEN: THE HEALTHY COMMUNITY COUNCIL 2011 ASSESSMENT}

doi:10.1136/jech.2011.142976i.87

${ }^{1} \mathrm{~K}$ Lewis, ${ }^{*} \mathrm{C}$ Reeves. ${ }^{1}$ James Madison University, Harrisonburg, Virginia, USA ${ }^{2}$ Rockingham Memorial Hospital, Harrisonburg, Virginia, USA

Introduction The Healthy Community Council (HCC) is a nonprofit community-based network composed of over 100 local stakeholders representing both public and private organisations within the community. The HCC has been working to improve the health of the community by conducting needs assessments, identifying priorities and assisting in the implementation of programs and 